\title{
Differential levels of L-homocysteic acid and lysophosphatidylcholine (16:0) in sera of patients with ovarian cancer
}

\author{
SEUNG CHEOL KIM $^{1 *}$, MIN KYUNG KIM ${ }^{2 *}$, YUN HWAN KIM ${ }^{1 *}$, SUN-A AHN $^{2}$, KYUNG-HEE KIM $^{2}$, \\ $\mathrm{KUN} \mathrm{KIM}^{2}$, WON KI KIM ${ }^{2}$, JUN HWA LEE ${ }^{1}$, JAE YOUL $\mathrm{CHO}^{3}$ and BYONG CHUL YOO ${ }^{2}$
}

${ }^{1}$ Division of Gynecologic Oncology, Department of Obstetrics and Gynecology, Ewha Woman's University Mokdong Hospital, Ewha Woman's University School of Medicine, Seoul 158-710; ${ }^{2}$ Colorectal Cancer Branch, Research Institute, National Cancer Center, Gyeonggi 410-769; ${ }^{3}$ Department of Genetic Engineering, Sungkyunkwan University, Gyeonggi 440-746, Republic of Korea

Received December 2, 2013; Accepted April 16, 2014

DOI: $10.3892 / 01.2014 .2214$

\begin{abstract}
Ovarian cancer (OVC) is one of the most difficult types of cancer to detect in the early stages of its development. There have been numerous attempts to identify a biomarker for OVC; however, an accurate diagnostic marker has yet to be identified. The present study profiled OVC candidate metabolites from the serum to identify potential diagnostic markers for OVC. Data regarding low-mass ions (LMIs) in the serum were obtained using matrix-assisted laser desorption/ionization (MALDI)-time-of-flight analysis. MALDI-mass spectrometry (MS) analysis of each serum sample was repeated six times in order to reduce the likelihood of experimental errors. The intensity of the LMI mass peaks were normalized using total peak area sums. The normalized intensity of LMI was used in principal component analysis-discriminant analysis to differentiate between 142 patients with OVC and 100 healthy control participants. Liquid chromatography-MS/MS was used to identify the selected LMIs. Extracted ion chromatogram analysis was used to measure the relative quantity of candidate metabolites from the LMI mass peak areas. The concentration of common metabolites in the serum was determined using ELISA. The top 20 LMI mass peaks with a weigh factor over 0.05 were selected to distinguish between the patients with OVC and the controls. Among the LMIs, two with 184.05 and $496.30 \mathrm{~m} / \mathrm{z}$ were identified as L-homocysteic acid (HCA) and lysophosphatidylcholine (LPC) (16:0), respectively. The
\end{abstract}

Correspondence to: Dr Byong Chul Yoo, Colorectal Cancer Branch, Research Institute, National Cancer Center, 323 Ilsanro, Goyang, Gyeonggi 410-769, Republic of Korea

E-mail: yoo_akh@ncc.re.kr

${ }^{*}$ Contributed equally

Key words: ovarian cancer, L-homocysteic acid, lysophosphatidylcholine (16:0), biomarker, cancer screening relative quantity of LPC (16:0) was found to be decreased in the OVC serum $(\mathrm{P}=0.05)$, while the quantity of HCA was observed to be significantly higher in the OVC serum $(\mathrm{P}<0.001)$. HCA was not detected in 59 cases out of the 63 control participants; however, the majority of the cases of OVC (16/25) exhibited significantly higher quantities of HCA. When the cutoff was $10 \mathrm{nmol} / \mathrm{ml}$, the sensitivity and specificity of HCA were 64.0 and $96.9 \%$, respectively. The level of LPC (16:0) was significantly correlated with tumor grade $(\mathrm{P}=0.045)$. HCA and LPC (16:0) showed correlation with stage and tumor histology, but the limited sample size resulted in a lack of statistical significance. The findings of the present study suggest that HCA may have potential to be a biomarker for OVC. The stratified screening including LPC (16:0) did not significantly increase the power for OVC screening; however, the present study showed that profiling LMIs in serum may be useful for identifying candidate metabolites for OVC screening.

\section{Introduction}

Ovarian cancer (OVC) is one of the most frequently occurring types of gynecological cancer, with 204,000 new cases identified each year and a five-year survival rate of $44 \%$ for all stages of cancer development (1-4). More than $70 \%$ of OVC cases are identified in the late stages of cancer (stage III or IV according to the International Federation of Gynecology and Obstetrics standard) (2). Despite improvements in anticancer therapeutic methods, the mortality rate of OVC has not decreased over the past 20 years due to difficulties in screening early stages of the disease (5). Current diagnostic methods include pelvic examination, ultrasonograms, blood tests and tissue examination $(6,7)$; however, these methods have several limitations, including their inability to diagnose OVC at an early stage or to detect invasiveness. Thus, early and easy-to-use diagnostic methods for OVC are required in order to increase the survival rate of patients with OVC.

Several previous studies have investigated the use of serological markers to accurately detect OVC. Such markers include cancer antigen (CA) 125, human epididymis 
protein 4 (HE4), and macrophage colony-stimulating factor (M-CSF) $(5,8,10)$. Serum CA125 and HE4 concentrations have been used as markers for OVC using radioimmunoassay $(6,9,10)$. Furthermore, $70 \%$ of patients with OVC with various OVC cell lines have high serum levels of M-CSF (11). While M-CSF is a monocyte-specific cytokine for proliferation and differentiation, it also acts as a growth factor for certain epithelial cancers in an autocrine and paracrine manner (12). However, these markers lack accuracy and have difficulty in early diagnosis. For example CA125 was discovered 20 years ago and has been used widely as an OVC marker since (13). However, CA125 has low specificity and sensitivity during the early stages of OVC $(6,9,14)$, thus an ideal marker has yet to be elucidated.

The present study profiled low-mass metabolic compounds in methanol/chloroform extracts obtained from the sera of patients with OVC and healthy controls using matrix-assisted laser desorption/ionization-time-of-flight (MALDI-TOF) mass spectrometry and identified two molecules using tandem mass spectrometry (MS/MS) analysis. The present study identified a differential pattern of lysophosphatidylcholine (LPC) (16:0) and L-homocysteic acid (HCA) in patients with OVC, and discusses the advantages of profiling low-mass metabolic compounds for screening OVC.

\section{Materials and methods}

Serum from patients with $O V C$. All participants provided written informed consent and the study protocol was approved by the Institutional Review Board of the Ewha Womans University (Seoul, Korea). A total of 142 patients and 100 control participants were enrolled in the present study (Table I).

MALDI-TOF analysis for collecting low-mass ions (LMIs) in serum. Four times volume of methanol/chloroform $(2: 1 ; \mathrm{v} / \mathrm{v})$ was incubated with $25 \mu \mathrm{l}$ serum for $10 \mathrm{~min}$ at room temperature subsequent to vortexing. The solution was centrifuged at $6,000 \mathrm{x} \mathrm{g}$ for $10 \mathrm{~min}$ at $4^{\circ} \mathrm{C}$. The supernatant was then dried in a concentrator for $1 \mathrm{~h}$ and resolved in $30 \mu \mathrm{l} 50 \%$ acetonitrile/ $0.1 \%$ trifluoroacetic acid (TFA) using a vortex for $30 \mathrm{~min}$.

Methanol/chloroform extract was mixed $(1: 12 ; \mathrm{v} / \mathrm{v})$ with an $\alpha$-cyano-4-hydroxycinnamic acid solution in $50 \%$ acetonitrile/ $0.1 \%$ TFA. A total of $1 \mu 1$ of the solution was then spotted on the MALDI target for analysis. Individual mass spectra from the serum extracts of the patients with OVC were obtained using a 4700 Proteomics Analyzer (Ab Sciex, Framingham, MA, USA). The mass-spectral data represent the average of 20 accumulated spectra. All individual peak areas were normalized to the total area up to $2,500 \mathrm{~m} / \mathrm{z}$. To minimize experimental error, variable factors including focus mass, laser intensity, target plate and data acquisition time were tested. The ideal focus mass and laser intensity were fixed at $500 \mathrm{~m} / \mathrm{z}$ and 5,000, respectively (15). With the fixed focus mass and laser intensity, one sample was analyzed six times under the different extraction and data acquisition times.

LMI selection and statistical analysis. All MALDI mass spectra, formatted as $\mathrm{t} 2 \mathrm{~d}$ files, were analyzed
Table I. Characteristics of the patients with ovarian cancer and the control participants included in the present study.

\begin{tabular}{|c|c|c|}
\hline Parameter & $\begin{array}{l}\text { Ovarian cancer } \\
\qquad(n=142)\end{array}$ & $\begin{array}{l}\text { Control } \\
(n=100)\end{array}$ \\
\hline Age, mean $\pm \mathrm{SD}$ & $52 \pm 13$ & $51 \pm 13$ \\
\hline \multicolumn{3}{|l|}{ Stage, n (\%) } \\
\hline IA & $37(26.1)$ & - \\
\hline IB & $2(1.4)$ & - \\
\hline $\mathrm{IC}$ & $12(8.5)$ & - \\
\hline IIA & $0(0.0)$ & - \\
\hline IIB & $1(0.7)$ & - \\
\hline IIIA & $0(0.0)$ & - \\
\hline IIIB & $1(0.7)$ & - \\
\hline IIIC & $77(54.2)$ & - \\
\hline IV & $12(8.5)$ & - \\
\hline \multicolumn{3}{|l|}{ Histology, n (\%) } \\
\hline Serous & $90(63.4)$ & - \\
\hline Mucinous & $23(16.2)$ & - \\
\hline Endometrioid & $8(5.6)$ & - \\
\hline Clear cell & $11(7.7)$ & - \\
\hline Transitional cell & $7(4.9)$ & - \\
\hline Mixed & $3(2.1)$ & - \\
\hline \multicolumn{3}{|l|}{ Grade, n (\%) } \\
\hline Mild & $24(16.9)$ & - \\
\hline Moderate & $35(24.6)$ & - \\
\hline Severe & $83(58.5)$ & - \\
\hline
\end{tabular}

SD, standard deviation.

using MarkerView ${ }^{\mathrm{TM}}$ software, version 1.2 (Applied $^{-}$ Biosystems/MDS Sciex, Toronto, ON, Canada). The optimized parameters used to compare LMI mass peaks in the serum extracts obtained from the patients with OVC were as follows: Mass tolerance, $100 \mathrm{ppm}$; minimum required response, 100; maximum number of peaks, 5000; and normalization, by total area sums. Subsequent to collecting the data using MALDI mass spectra, principal component analysis-discriminant analysis (PCA-DA) and t-tests were used to select LMIs with differential peak intensities in serum extracts from patients with OVC.

Measurement of HCA in serum. The level of HCA in the sera was measured using an ELISA kit (Cusabio Biotech, Co., Ltd., Wuhan, China) according to the manufacturer's instructions.

Measurement of LPC (16:0) in serum. A nanoflow high-performance liquid chromatography instrument (Easy nLC; Thermo Scientific, Inc., Waltham, MA, USA) was coupled to an LTQ mass spectrometer (Thermo Scientific, Inc.). A PepMap ${ }^{\circledR}$ RSLC, C18, $2 \mu \mathrm{m}, 100 \AA$ analytical column $(50 \mathrm{~cm}$; inner diameter, $75 \mu \mathrm{m}$; Dianex Corporation, Sunnyvale, CA, USA) was used. Reversed phase chromatography was performed using a binary buffer system consisting of $0.1 \%$ formic acid 

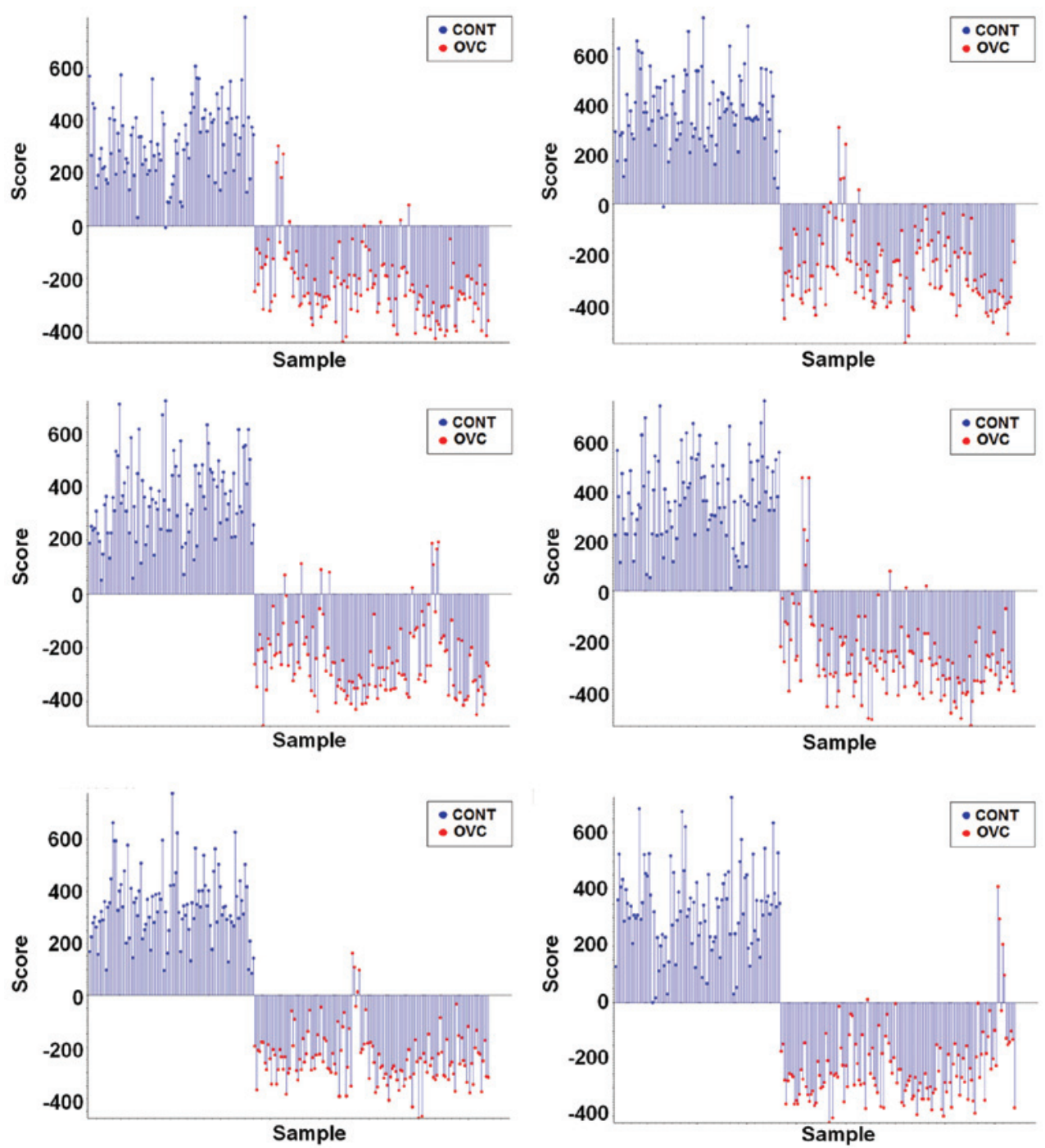

Figure 1. PCA-DA of the sera of 142 patients with OVC and 100 healthy controls. Methanol/chloroform extracts from the sera of 142 patients with OVC and 100 healthy controls were used for MALDI-TOF analysis. LMI data ( $\mathrm{m} / \mathrm{z}$ and mass peak intensity) from the extracts was obtained using MALDI-TOF analysis six times. The intensities of all of the individual LMIs were normalized using the 'total peak area sums'. The $\mathrm{m} / \mathrm{z}$ and normalized intensity of LMI was used in PCA-DA. Classification results of PCA-DA repeated six times reveal that the pattern of LMI in the sera of the patients with OVC was different to that of the controls. PCA-DA, principal component analysis-discriminant analysis; OVC, ovarian cancer; MALDI-TOF, matrix-assisted laser desorption/ionization-time-of-flight; LMI, low-mass ion; Cont, control.

(buffer A) and acetonitrile in $0.1 \%$ formic acid (buffer B). The sample was separated using a linear gradient of $3-50 \%$ buffer $\mathrm{B}$ at a flow rate of $300 \mathrm{nl} / \mathrm{min}$. The gradient time was $90 \mathrm{~min}$ and the total run time for the liquid chromatography MS/MS was $120 \mathrm{~min}$. The extracted LPC was analyzed using the selected reaction monitoring (SRM) mode. The SRM transitions for the LPC lipid were set to $\mathrm{m} / \mathrm{z} 496.4$ to 183.96 and $\mathrm{m} / \mathrm{z} 496.4$ to 478.33 . The SRM data were acquired within fragment ion mass $\pm 2 \mathrm{~m} / \mathrm{z}$ and each SRM transition and respective retention time was validated for specific LPC. Data were processed through integrating the appropriate peaks for LPC, followed by comparing the calculated peak areas using two-paired t-tests.

Statistical analysis. Between-group differences were calculated using the student's t-test and within-group correlations were calculated using Spearman's rank correlation coefficient. $\mathrm{P}<0.05$ was considered to indicate a statistically significant difference.

\section{Results}

Differential LMIs in methanol/chloroform extracts from the sera of patients with OVC. Data ( $\mathrm{m} / \mathrm{z}$ and mass peak intensity) regarding the LMIs with mostly $<1,000 \mathrm{~m} / \mathrm{z}$ collected from the sera extracts of 100 healthy control individuals and 142 patients with OVC were used in the PCA-DA in order to determine whether differential LMI patterns exist in the sera of patients with OVC. Supervised PCA-DA using LMI data obtained from six repeats of MALDI-TOF analysis discriminated the patients with OVC from the control individuals (Fig. 1).

Selection and identification of LMIs showing a differential pattern in patients with OVC. Weighting factors (loading value) for all individual LMIs were calculated using PCA-DA (Fig. 2). LMIs which consistently exhibited higher weighting factors in six different PCA-DA analyses were selected. Despite 
Table II. Candidate metabolites with $\mathrm{H}^{+}$adducts in human metabolome database.

\begin{tabular}{|c|c|c|c|c|c|}
\hline Compound & Name & Adduct & $\begin{array}{l}\text { Adduct } \\
\text { MW (Da) }\end{array}$ & $\begin{array}{l}\text { Compound } \\
\text { MW (Da) }\end{array}$ & Delta \\
\hline \multicolumn{6}{|c|}{ Metabolites with $184.05 \pm 0.05 \mathrm{~m} / \mathrm{z}$} \\
\hline HMDB00017 & 4-Pyridoxic acid & $\mathrm{M}+\mathrm{H}$ & 184.06043 & 183.05316 & 0.010434 \\
\hline HMDB02205 & L-Homocysteic acid & $\mathrm{M}+\mathrm{H}$ & 184.02742 & 183.02014 & 0.022581 \\
\hline HMDB11657 & $\begin{array}{l}\text { 2,6-Diamino-4-hydroxy-5-N- } \\
\text { ethylformamidopyrimidine }\end{array}$ & $\mathrm{M}+\mathrm{H}$ & 184.08290 & 183.07563 & 0.032901 \\
\hline HMDB33141 & 2-Amino- $\alpha$-carboline & $\mathrm{M}+\mathrm{H}$ & 184.08692 & 183.07965 & 0.036923 \\
\hline HMDB29723 & Saccharin & $\mathrm{M}+\mathrm{H}$ & 184.00629 & 182.99901 & 0.043710 \\
\hline HMDB02832 & Methylnoradrenaline & $\mathrm{M}+\mathrm{H}$ & 184.09682 & 183.08954 & 0.046819 \\
\hline HMDB15652 & Levonordefrin & $\mathrm{M}+\mathrm{H}$ & 184.09682 & 183.08954 & 0.046819 \\
\hline HMDB00819 & Normetanephrine & $\mathrm{M}+\mathrm{H}$ & 184.09682 & 183.08954 & 0.046819 \\
\hline HMDB29455 & Ginkgotoxin & $\mathrm{M}+\mathrm{H}$ & 184.09682 & 183.08954 & 0.046819 \\
\hline HMDB00068 & Epinephrine & $\mathrm{M}+\mathrm{H}$ & 184.09682 & 183.08954 & 0.046819 \\
\hline \multicolumn{6}{|c|}{ Metabolites with $496.30 \pm 0.05 \mathrm{~m} / \mathrm{z}$} \\
\hline HMDB10382 & LPC (16:0) & $\mathrm{M}+\mathrm{H}$ & 496.33977 & 495.33249 & 0.039765 \\
\hline
\end{tabular}

LPC, lysophosphatidylcholine.
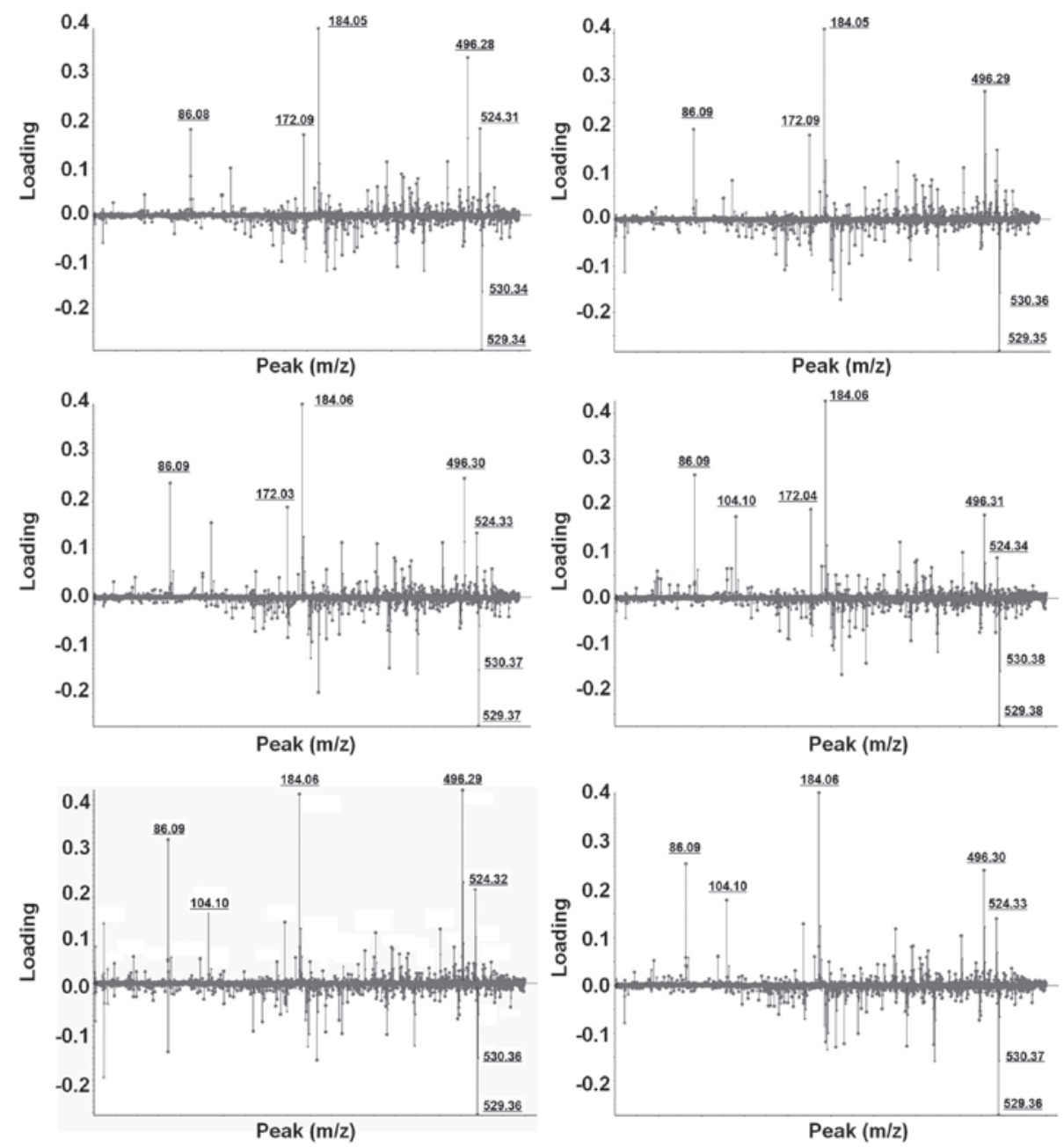

Figure 2. Selection of LMIs with higher weighting factors. Weighting factors (or loading) for individual LMIs were calculated using principal component analysis-discriminant analysis. LMIs showing a higher weighting factor in each independent analysis were selected for further identification and validation. There was a slight mass shifting in repeated matrix-assisted laser desorption/ionization-time-of-flight analyses. LMIs with 184.05 and $496.30 \mathrm{~m} / \mathrm{z}$ showed strong and constant discriminating power for ovarian cancer screening. LMI, low-mass ion. 
Table III. L-homocysteic acid levels in the sera of 63 control participants and 25 patients with OVC.

\begin{tabular}{|c|c|c|c|c|c|c|c|}
\hline \multicolumn{6}{|c|}{ Control } & \multicolumn{2}{|c|}{ OVC } \\
\hline Sample no. & $\begin{array}{c}\text { Conc. } \\
(\mathrm{nmol} / \mathrm{ml})\end{array}$ & Sample no. & $\begin{array}{c}\text { Conc. } \\
(\mathrm{nmol} / \mathrm{ml})\end{array}$ & Sample no. & $\begin{array}{c}\text { Conc. } \\
(\mathrm{nmol} / \mathrm{ml})\end{array}$ & Sample no. & $\begin{array}{c}\text { Conc. } \\
(\mathrm{nmol} / \mathrm{ml})\end{array}$ \\
\hline Control 01 & 0.000 & Control 26 & 0.000 & Control 51 & 0.000 & OVC 01 & 25.991 \\
\hline Control 02 & 0.000 & Control 27 & 0.000 & Control 52 & 0.000 & OVC 02 & 0.000 \\
\hline Control 03 & 0.000 & Control 28 & 0.000 & Control 53 & 48.750 & OVC 03 & 109.620 \\
\hline Control 04 & 0.000 & Control 29 & 0.000 & Control 54 & 0.000 & OVC 04 & 0.000 \\
\hline Control 05 & 0.000 & Control 30 & 0.000 & Control 55 & 0.000 & OVC 05 & 20.037 \\
\hline Control 06 & 0.000 & Control 31 & 0.000 & Control 56 & 0.000 & OVC 06 & 0.000 \\
\hline Control 07 & 0.000 & Control 32 & 0.000 & Control 57 & 0.000 & OVC 07 & 0.000 \\
\hline Control 08 & 0.000 & Control 33 & 0.000 & Control 58 & 0.000 & OVC 08 & 0.000 \\
\hline Control 09 & 0.000 & Control 34 & 0.000 & Control 59 & 0.000 & OVC 09 & 116.759 \\
\hline Control 10 & 0.000 & Control 35 & 0.000 & Control 60 & 0.000 & OVC 10 & 79.676 \\
\hline Control 11 & 0.000 & Control 36 & 0.111 & Control 61 & 0.000 & OVC 11 & 61.083 \\
\hline Control 12 & 0.000 & Control 37 & 0.000 & Control 62 & 0.000 & OVC 12 & 172.352 \\
\hline Control 13 & 0.000 & Control 38 & 0.000 & Control 63 & 0.000 & OVC 13 & 286.398 \\
\hline Control 14 & 0.000 & Control 39 & 0.000 & & & OVC 14 & 203.306 \\
\hline Control 15 & 0.000 & Control 40 & 0.000 & & & OVC 15 & 0.000 \\
\hline Control 16 & 0.000 & Control 41 & 0.000 & & & OVC 16 & 175.713 \\
\hline Control 17 & 0.000 & Control 42 & 0.000 & & & OVC 17 & 175.676 \\
\hline Control 18 & 0.000 & Control 43 & 0.000 & & & OVC 18 & 74.824 \\
\hline Control 19 & 4.981 & Control 44 & 0.000 & & & OVC 19 & 133.407 \\
\hline Control 20 & 0.000 & Control 45 & 49.750 & & & OVC 20 & 344.787 \\
\hline Control 21 & 0.000 & Control 46 & 0.000 & & & OVC 21 & 206.537 \\
\hline Control 22 & 0.000 & Control 47 & 0.000 & & & OVC 22 & 0.000 \\
\hline Control 23 & 0.000 & Control 48 & 0.000 & & & OVC 23 & 0.000 \\
\hline Control 24 & 0.000 & Control 49 & 0.000 & & & OVC 24 & 72.565 \\
\hline Control 25 & 0.000 & Control 50 & 0.000 & & & OVC 25 & 0.000 \\
\hline
\end{tabular}

OVC, ovarian cancer; Conc., concentration.

slight mass shifting, LMIs with 184.05 and $496.30 \mathrm{~m} / \mathrm{z}$ showed strong discriminating power for OVC screening (Fig. 2).

In order to identify LMIs with 184.05 and $496.30 \mathrm{~m} / \mathrm{z}$, candidate metabolites within $\pm 0.05 \mathrm{~m} / \mathrm{z}$ difference were identified using the Human Metabolome Database (HMDB). Ten candidate metabolites with $184.05 \pm 0.05 \mathrm{~m} / \mathrm{z}$ were identified (Table II). Among the candidate metabolites, the metabolic description of HCA in the HMDB was most correlated with OVC, and LPC (16:0) was the only metabolite with $496.30 \pm 0.05 \mathrm{~m} / \mathrm{z}$ (Table II). The LMI with $496.30 \mathrm{~m} / \mathrm{z}$ on the mass spectrum (Fig. 3A) was further analyzed using MS/MS analysis and was identified to be LPC (16:0) through comparing the MS/MS spectrum of lipid compounds (Fig. 3B).

Differential level of HCA and LPC (16:0). The level of HCA was assessed in 63 control participants and 25 patients with OVC (Table III). Due to insufficient amounts of sera, HCA was not detected in 59/63 of the controls, but the majority of cases of OVC (16/25) exhibited significantly higher levels of HCA, with the mean HCA concentration in the sera of the control individuals being $0.16 \mathrm{nmol} / \mathrm{ml}$ compared with $0.60 \mathrm{nmol} / \mathrm{ml}$ in the patients with OVC $(\mathrm{P}<0.001$; Fig. 4A). At the cutoff of $10 \mathrm{nmol} / \mathrm{ml}$, the sensitivity and specificity of HCA were 64.0 and $96.9 \%$, respectively; thus, HCA may have potential for OVC screening (Table III).

LPC (16:0) was detected as an LMI with either 183.96 or $478.33 \mathrm{~m} / \mathrm{z}$ in LC-MS/MS analysis (Fig. 5A). A sufficient amount of sera was obtained from 19 control individuals and 20 patients with OVC to quantify the level of LPC (16:0) and peak areas of 183.96 and $478.33 \mathrm{~m} / \mathrm{z}$ were determined (Table IV). The peak area was variable depending on the individual samples, but the level of LPC (16:0), represented by peak areas of 183.96 and $478.33 \mathrm{~m} / \mathrm{z}$, was observed to be lower in the sera of patients with OVC compared with that of the controls $(\mathrm{P}=0.0515$ and 0.0508 , respectively; Fig. 5B).

Clinicopathological relevance of $L P C$ (16:0) and HCA in $O V C$. Increased LPC (16:0) was found to be significantly correlated with tumor grade $(\mathrm{P}=0.045)$. Although not statistically significant, possibly due to the small number of samples, HCA and LPC (16:0) were found to be correlated with stage and tumor histology (data not shown). 
Table IV. Level of peak area in the sera of 19 control participants and 25 patients with OVC.

\begin{tabular}{|c|c|c|c|c|c|}
\hline \multicolumn{3}{|c|}{ Control } & \multicolumn{3}{|c|}{$\mathrm{OVC}$} \\
\hline Sample no. & $183.96 \mathrm{~m} / \mathrm{z}$ & $478.33 \mathrm{~m} / \mathrm{z}$ & Sample no. & $183.96 \mathrm{~m} / \mathrm{z}$ & $478.33 \mathrm{~m} / \mathrm{z}$ \\
\hline Control 01 & 177013 & 376781 & OVC 01 & 14153 & 30741 \\
\hline Control 02 & 69756 & 137502 & OVC 02 & 5859 & 13745 \\
\hline Control 03 & 124532 & 272566 & OVC 03 & 7971 & 15462 \\
\hline Control 05 & 25420 & 66997 & OVC 04 & 8396 & 19590 \\
\hline Control 06 & 54801 & 128622 & OVC 05 & 20228 & 53475 \\
\hline Control 07 & 37451 & 84449 & OVC 06 & 16271 & 39552 \\
\hline Control 08 & 83913 & 172936 & OVC 07 & 32559 & 69217 \\
\hline Control 09 & 24680 & 64998 & OVC 08 & 25213 & 60890 \\
\hline Control 10 & 24203 & 53327 & OVC 09 & 12003 & 30286 \\
\hline Control 11 & 154157 & 376840 & OVC 10 & 26121 & 59037 \\
\hline Control 12 & 22627 & 51433 & OVC 11 & 33905 & 51453 \\
\hline Control 13 & 48125 & 102808 & OVC 12 & 51453 & 115718 \\
\hline Control 14 & 52038 & 109721 & OVC 13 & 40846 & 87236 \\
\hline Control 15 & 45143 & 104486 & OVC 14 & 68258 & 149730 \\
\hline Control 16 & 10764 & 24637 & OVC 15 & 49476 & 114076 \\
\hline Control 17 & 4301 & 10910 & OVC 16 & 53280 & 132663 \\
\hline Control 18 & 6538 & 15969 & OVC 17 & 49151 & 109521 \\
\hline Control 19 & 5664 & 8595 & OVC 18 & 40636 & 84156 \\
\hline \multirow[t]{7}{*}{ Control 20} & 1894 & 3979 & OVC 19 & 35516 & 78462 \\
\hline & & & OVC 20 & 59084 & 130141 \\
\hline & & & OVC 21 & 38533 & 80979 \\
\hline & & & OVC 22 & 14389 & 31289 \\
\hline & & & OVC 23 & 7779 & 15915 \\
\hline & & & OVC 24 & 35065 & 83313 \\
\hline & & & OVC 25 & 12879 & 29815 \\
\hline
\end{tabular}

OVC, ovarian cancer.

\section{Discussion}

Despite previous investigations, a diagnostic marker for the early diagnosis of OVC has yet to be elucidated. Previous markers which have been used for OVC, including CA125 and $\mathrm{HE} 4$, only detected $\mathrm{OVC}$ at the late stages of cancer development and lacked efficiency during early tumor growth $(13,14)$.

Metabolic compounds are detected as LMIs in mass spectrometry. Our previous study showed an example of LMI profiling for cancer screening (15). However, at present, the dynamic status of metabolic compounds in the blood is poorly understood. Metabolic compounds in the blood are capable of showing disease status; therefore, profiling LMIs may be useful not only for understanding cancer, but also for identifying biomarkers. Furthermore, recent mass technology, including MALDI-TOF and liquid chromatography-MS/MS, has been found to provide extremely precise and accurate data on LMIs. Therefore, the present study aimed to profile LMIs in serum extracts to assess whether such profiling is capable of discriminate OVC. PCA-DA results showed that the profile of LMIs discriminated OVC (Fig. 1). Only one control case was assigned as OVC over the six experimental repeats (Fig. 1), allowing the LMIs with a significant effect of discriminating OVC to be selected (Fig. 2). Two metabolic compounds were identified and quantified: HCA and LPC (16:0) (Figs. 3-5).

HCA has been reported to affect the oxidation of homocysteinethiolactone to sulfated glycosaminoglycans in cartilage (16). The free base of homocysteinethiolactone has been found to induce carcinogenesis in a mouse model, thus abnormal homocysteine metabolism may be associated with carcinogenesis (16). Dysregulated levels of HCA have not been reported in cancers, although markedly increased HCA has been detected in the cerebrospinal fluid of patients with lymphoma treated with methtrexate $(17,18)$. In the present study, the profiling of LMIs revealed that the level of HCA was different in the serum of patients with OVC compared with healthy control individuals, which was shown through the quantification of HCA in the sera of the controls and the patients with OVC (Fig. 4 and Table III). HCA was not detected in the majority of the control participants, but many of the patients with OVC (16/25) showed significantly higher HCA levels (Table III). At the cutoff of $10 \mathrm{nmol} / \mathrm{ml}$, the sensitivity and specificity of HCA were 64.0 and $96.9 \%$, respectively. 
A

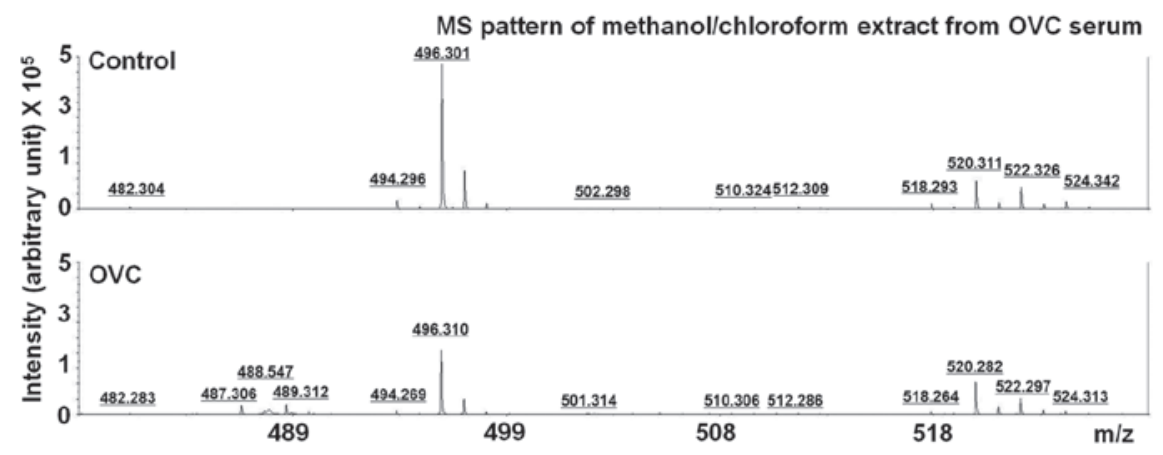

B

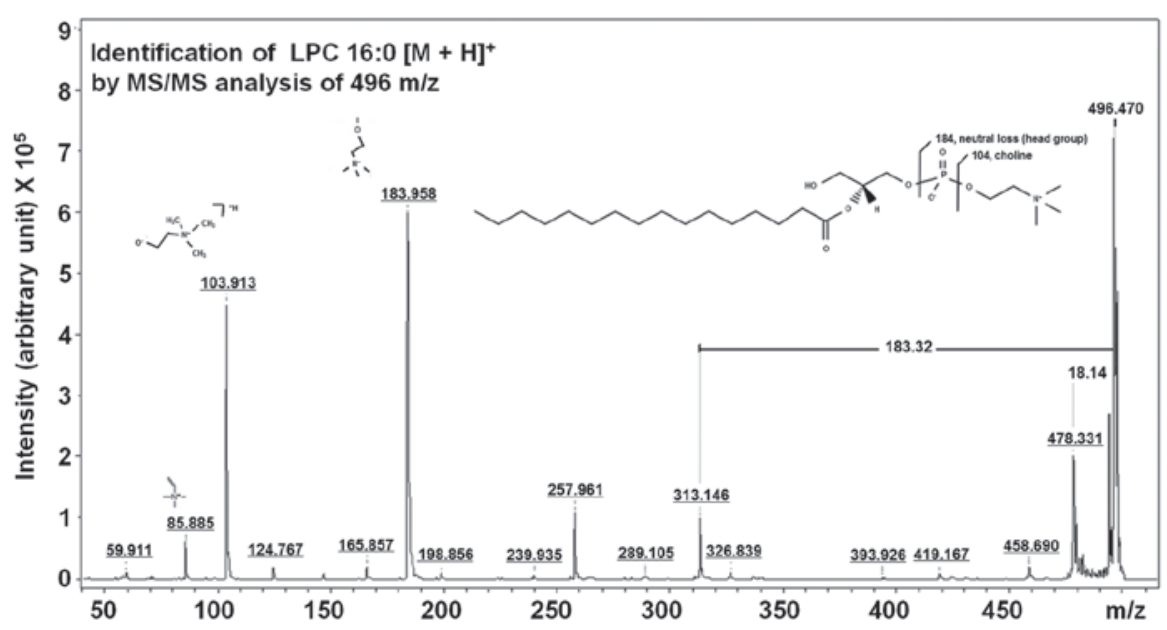

Figure 3. Identification of LMIs with $496.30 \mathrm{~m} / \mathrm{z}$. (A) Mass peak pattern of an LMI with $496.30 \mathrm{~m} / \mathrm{z}$ on mass spectra. (B) MS/MS analysis for the identification of an LMI with $496.30 \mathrm{~m} / \mathrm{z}$. The MS/MS spectrum of the LMI with $496.30 \mathrm{~m} / \mathrm{z}$ was identical to that of LPC (16:0). LMI, low-mass ion; MS/MS, tandem mass spectrometry; LPC, lysophosphatidylcholine; OVC, ovarian cancer.

A

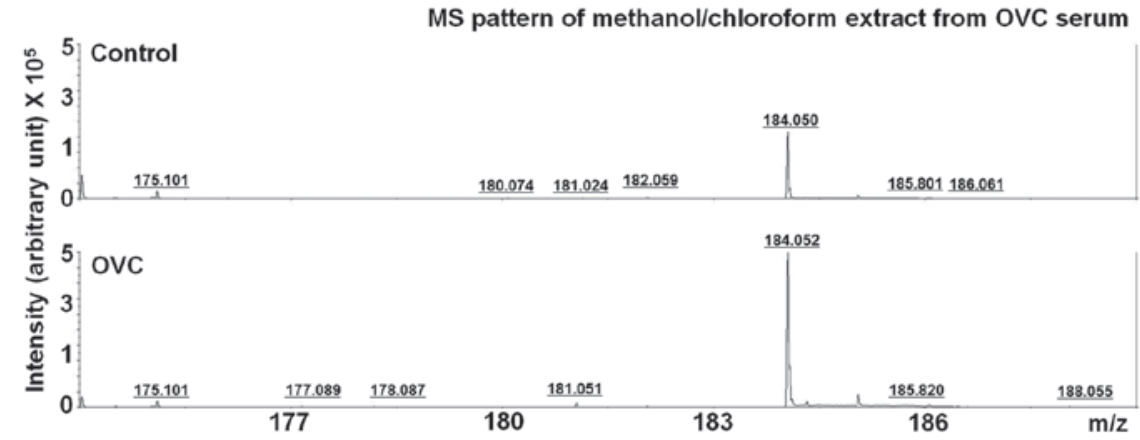

B

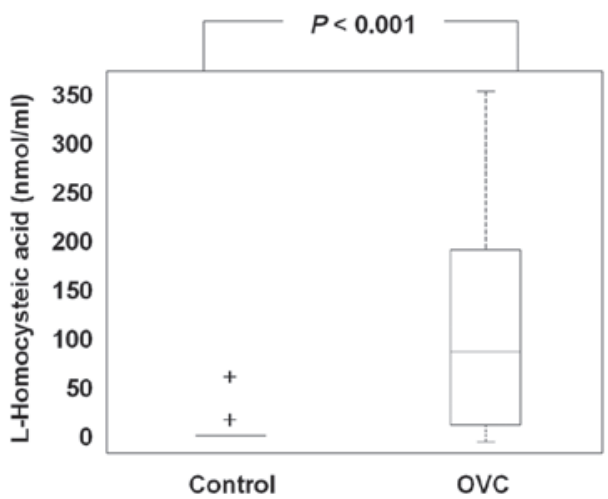

Figure 4. HCA is significantly increased in the sera of the patients with OVC. (A) Mass peak pattern of a low-mass ion with $184.05 \mathrm{~m} / \mathrm{z}$ on mass spectra. (B) Increase in HCA in patients with OVC compared with the control participants. HCA was not detected in the majority of the control participants. HCA was found to be significantly increased in the sera of the patients with OVC (P<0.001). HCA, L-homocysteic acid; OVC, ovarian cancer; MS, mass spectrometry. 
A

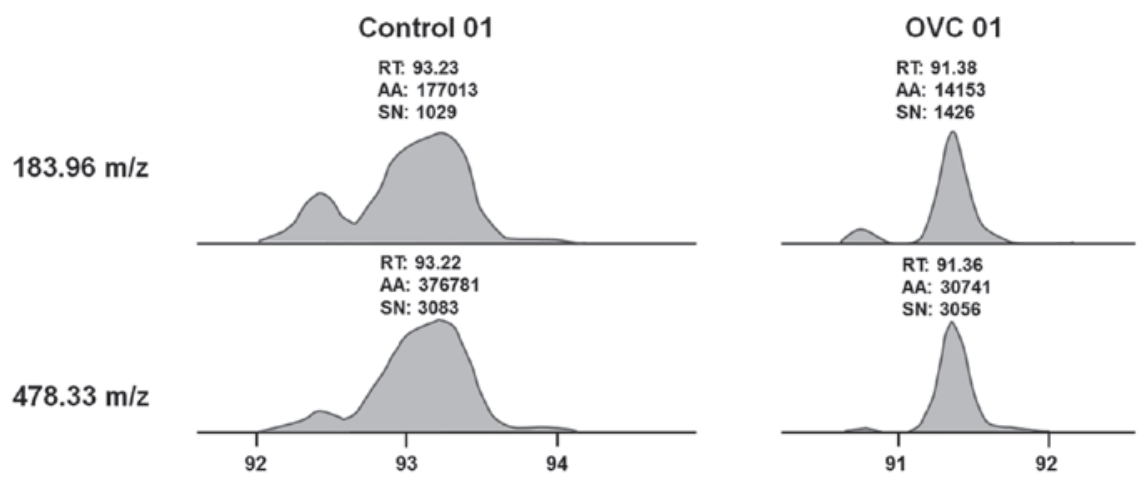

$\mathbf{B}$
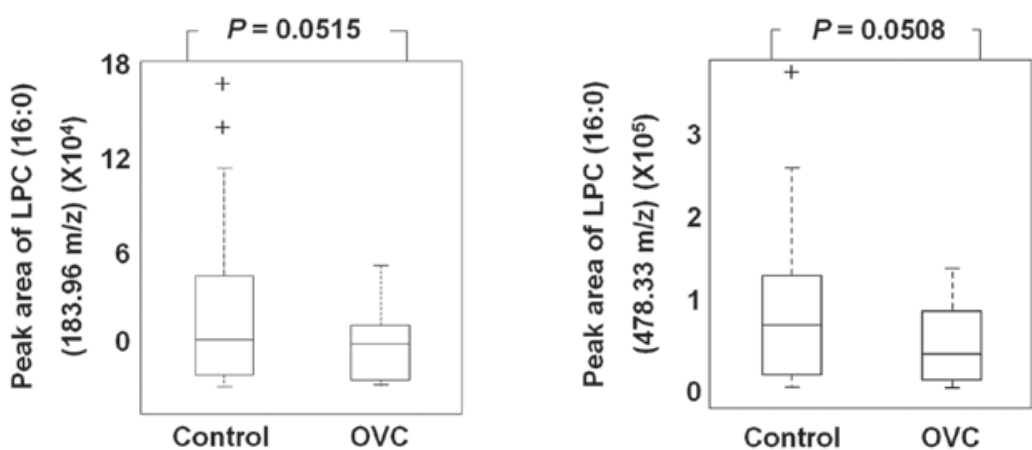

Figure 5. Decreased level of LPC (16:0) in the sera of patients with OVC. The level of LPC (16:0) was assessed through integrating the appropriate peaks for LPC, followed by calculating the ratio of the peak areas. (A) Extracted ion chromatogram of LPC (16:0) in the sera of the control participants and the patients with OVC. Peak areas at 183.96 and $478.33 \mathrm{~m} / \mathrm{z}$ indicated the concentration of LPC (16:0). (B) The peak area of LPC (16:0) at 183.96 and $478.33 \mathrm{~m} / \mathrm{z}$. The peak area of LPC (16:0) with either 183.96 or $478.33 \mathrm{~m} / \mathrm{z}$ was decreased in the sera of the patients with OVC compared with the controls, but was not significantly different ( $\mathrm{P}=0.0515$ and $\mathrm{P}=0.0508$, respectively). LPC, lysophosphatidylcholine; OVC, ovarian cancer; RT, retention time; AA, peak area counts; SN: signal-to-noise ratio.

The biological implications of upregulated HCA in the sera of patients with OVC has yet to be elucidated and the level of HCA in other types of cancer has yet to be reported. However, the present study found that HCA has strong potential for OVC screening.

The level of LPC in the blood of patients with cancer varies depending on the type of cancer, with LPC found to be decreased in breast cancer (19) and increased in hepatocellular carcinoma (20). In the present study, LPC (16:0) was observed to be decreased in the serum of patients with OVC (Fig. 5). LPC acts as a bioactive mediator in wound healing and inflammation (21), but also has a role in the progression of OVC (22) and lung cancer (23). LPC has many subtypes, and each subtype has a different length of carbon chain. Although the role of each LPC subtype has yet to be elucidated, in the present study, LPC (16:0) was found to be correlated with tumor grade in patients with $\mathrm{OVC}(\mathrm{P}=0.045)$.

In conclusion, the present study demonstrated that LMI profiling may be a powerful tool to obtain valuable data on metabolic compounds, as well as to identify biomarkers for cancer screening. Despite the lack of explanation for the pathological changes in HCA and LPC (16:0) in the sera of patients with OVC, the findings of the present study demonstrate that HCA is a powerful serological biomarker for OVC screening. In the present study, LPC alone was not helpful to increase the discriminating power of HCA; however, with the identification of other candidate metabolites in the future, HCA has the potential to be used in multi-biomarker OVC screening.

\section{Acknowledgements}

The present study was supported by a grant from the Korean Health Technology R\&D Project (Ministry of Health and Welfare, Korea; grant no. HI12C0050) and the Ewha Global Top 5 Grant 2011 of Ewha Womans University.

\section{References}

1. Toss A, De Matteis E, Rossi E, Casa LD, Iannone A, Federico M and Cortesi L: Ovarian cancer: can proteomics give new insights for therapy and diagnosis? Int J Mol Sci 14: 8271-8290, 2013.

2. Zhang B, Cai FF and Zhong XY: An overview of biomarkers for the ovarian cancer diagnosis. Eur J Obstet Gynecol Reprod Biol 158: 119-123, 2011.

3. Xu YZ, Xi QH, Ge WL and Zhang XQ: Identification of serum microRNA-21 as a biomarker for early detection and prognosis in human epithelial ovarian cancer. Asian Pac J Cancer Prev 14: 1057-1060, 2013.

4. Zhang B, Barekati Z, Kohler C, Radpour R, Asadollahi R, Holzgreve W and Zhong XY: Proteomics and biomarkers for ovarian cancer diagnosis. Ann Clin Lab Sci 40: 218-225, 2010.

5. Zhang Z, Bast RC Jr, Yu Y, et al: Three biomarkers identified from serum proteomic analysis for the detection of early stage ovarian cancer. Cancer Res 64: 5882-5890, 2004.

6. Jacobs I, Davies AP, Bridges J, et al: Prevalence screening for ovarian cancer in postmenopausal women by CA 125 measurement and ultrasonography. BMJ 306: 1030-1034, 1993.

7. Goswamy RK, Campbell S and Whitehead MI: Screening for ovarian cancer. Clin Obstet Gynaecol 10: 621-643, 1983.

8. Sarojini S, Tamir A, Lim H, et al: Early detection biomarkers for ovarian cancer. J Oncol 2012: 709049, 2012.

9. Jacobs IJ, Skates S, Davies AP, et al: Risk of diagnosis of ovarian cancer after raised serum CA 125 concentration: a prospective cohort study. BMJ 313: 1355-1358, 1996. 
10. Anderson GL, McIntosh M, Wu L, et al: Assessing lead time of selected ovarian cancer biomarkers: a nested case-control study. J Natl Cancer Inst 102: 26-38, 2010.

11. Bast RC Jr, Boyer CM, Jacobs I, et al: Cell growth regulation in epithelial ovarian cancer. Cancer 71 (4 Suppl): 1597-1601, 1993.

12. Moradi MM, Carson LF, Weinberg B, Haney AF, Twiggs LB and Ramakrishnan S: Serum and ascitic fluid levels of interleukin-1, interleukin-6, and tumor necrosis factor-alpha in patients with ovarian epithelial cancer. Cancer 72: 2433-2440, 1993.

13. Luo LY, Katsaros D, Scorilas A, et al: The serum concentration of human kallikrein 10 represents a novel biomarker for ovarian cancer diagnosis and prognosis. Cancer Res 63: 807-811, 2003.

14. Milojkovic M, Hrgovic Z, Hrgovic I, Jonat W, Maass N and Buković D: Significance of CA 125 serum level in discrimination between benign and malignant masses in the pelvis. Arch Gynecol Obstet 269: 176-180, 2004.

15. Yoo BC, Kong SY, Jang SG, et al: Identification of hypoxanthine as a urine marker for non-Hodgkin lymphoma by low-mass-ion profiling. BMC Cancer 10: 55, 2010.

16. McCully KS: Chemical pathology of homocysteine. III. Cellular function and aging. Ann Clin Lab Sci 24: 134-152, 1994.

17. Becker A, Vezmar S, Linnebank M, Pels H, Bode U, Schlegel U and Jaehde U: Marked elevation in homocysteine and homocysteine sulfinic acid in the cerebrospinal fluid of lymphoma patients receiving intensive treatment with methotrexate. Int J Clin Pharmacol Ther 45: 504-515, 2007.
18. Quinn CT, Griener JC, Bottiglieri T, Hyland K, Farrow A and Kamen BA: Elevation of homocysteine and excitatory amino acid neurotransmitters in the CSF of children who receive methotrexate for the treatment of cancer. J Clin Oncol 15: 2800-2806, 1997.

19. Qiu Y, Zhou B, Su M, et al: Mass spectrometry-based quantitative metabolomics revealed a distinct lipid profile in breast cancer patients. Int J Mol Sci 14: 8047-8061, 2013.

20. Ressom HW, Xiao JF, Tuli L, et al: Utilization of metabolomics to identify serum biomarkers for hepatocellular carcinoma in patients with liver cirrhosis. Anal Chim Acta 743: 90-100, 2012.

21. SakaiM,MiyazakiA,HakamataH,etal:Lysophosphatidylcholine plays an essential role in the mitogenic effect of oxidized low density lipoprotein on murine macrophages. J Biol Chem 269 31430-31435, 1994.

22. Fang X, Gaudette D, Furui T, et al: Lysophospholipid growth factors in the initiation, progression, metastases, and management of ovarian cancer. Ann NY Acad Sci 905: 188-208, 2000

23. Guo Y, Wang X, Qiu L, et al: Probing gender-specific lipid metabolites and diagnostic biomarkers for lung cancer using Fourier transform ion cyclotron resonance mass spectrometry. Clin Chim Acta 414: 135-141, 2012. 